\title{
TOWARDS A GEOGRAPHY OF NEPALESE CUISINE
}

\section{David Seddon ${ }^{1}$}

\begin{abstract}
This is the first part of an article which proposes to break new ground in developing a conceptual framework for and presenting a preliminary empirical analysis of 'the geography of Nepalese cuisine'. This part of the article sets out some of the elements required for an exploration of national, regional and local cuisines. It elaborates the concept of 'cuisine' as a historical but constantly evolving socio-economic and cultural construct (a food tradition) within a more-or-less defined geographical area. It considers the significance of 'food availability' and the ways in which the 'natural' world is classified and categorized to define what is considered edible and what is not. It explains how food preparation and processing transforms an animal, fish or plant into a food stuff or ingredient and examines how food preparation, production (including cooking) and presentation may differ and may be associated with different styles of cuisine (high and low, complex and simple, etc.). It introduces a distinction between national, regional and local cuisines and briefly considers the treatment of 'Indian' cuisine.
\end{abstract}

Key Words: Cuisine, Food, Food tradition, Nepal, Nepalese, Newari, Dalbhat

\section{Introduction}

It is said that 'fools rush in where angels fear to tread'. This article represents an example of just such a foolish initiative. I propose to initiate what I believe to be a new focus in the broad field of the Geography of Nepal.

Much has been written about the role of agriculture in the Nepalese economy; and something, although much less, has been written about the history and development of agriculture. There has been a continual concern, from the 1950s onwards, if not before, with issues of food production and food security, including, particularly among the most practical agencies associated with issues of food security (such as the World Food Programme), with the geography of food security. Economists have been interested for many years in patterns of trade both within the country and between Nepal and the outside world, and to some extent in the commodity chains associated with food stuffs and with other goods.

Social anthropologists have written extensively about both the daily lives and the cultural traditions of the various different ethnic groups and castes that constitute Nepalese society, in some cases referring to the crops they grow, the livestock they produce, and the foodstuffs they buy and sell and consume, and in some cases to the ceremonial, religious and other ritual occasions on which they perform various rites and consumer various foods and beverages. Claude Levi-Strauss has, famously, written about the symbolic and structural significance of food in his book The Raw and the Cooked (Le Cru et le Cuit) and influenced several other social anthropologists, such as Mary Douglas and Edmund Leach, to write about the logic of food taboos.

But to my knowledge, no-one has attempted, to develop a systematic approach to the geography of food and, more particularly, to the geography of Nepalese cuisine. Before I venture into this new field (in part two of this article), let me first (in part one) clarify a number of concepts and issues relevant to the development of a 'geography of cuisine' or food traditions.

\section{PART ONE}

\section{'Cuisine'}

By 'cuisine' I understand a distinctive sets of beliefs and practices surrounding the preparation, production, presentation and

\footnotetext{
${ }^{1}$ David Seddon was, until taking early retirement in 2006, Professor of Development Studies at the University of East Anglia in the UK. He is now Director of an independent consultancy company, Critical Faculty, and senior research associate at the Commonwealth Studies Policy Unit, attached to the Commonwealth Studies Institute, University of London. E mail criticalfaculty@ hotmail.com
} 
consumption of food, involving distinctive styles and processes of preparation and production of food (and drink), various distinctive forms of presentation of food and drink, and various distinctive patterns of consumption of food and drink. Some writers have used the term 'kitchen' in somewhat the same way, and indeed 'kitchen' is a legitimate translation of the French word 'cuisine'; but in English, the word 'kitchen' has more of the specific connotations of the place in which food preparation and cooking takes place (eg 'if you can't take the heat, get out of the kitchen').

The extent to which the existence of distinct national, regional or local 'cuisines' are 'recognized' varies considerably, largely by virtue of the extent to which they are defined and identified as such, whether by outsiders or by those involved. In other words, a national, regional or local 'cuisine' may be clearly and fully identified, vaguely discerned and described, or not defined at all. On the other hand, all societies have a distinctive set of beliefs and practices relating to food, its preparation, its production, presentation and consumption. 'Cuisines' are therefore social and cultural constructs, whose definition and characteristics are subject to change through time, both as a result of historical trends and processes, and also through conscious action and intervention. They are constantly being invented and reinvented, although some more rapidly and consciously than others.

Cuisines are to a considerable extent defined by the basic ingredients used in food and drink preparation, and these in turn depend on the availability of specific flora and fauna within a given territory or space - the environment. In so far as food traditions tend to develop within a more or less defined environment, we may speak reasonably of a geography of cuisine In so far as both flora and fauna themselves evolve and change, or are changed by human agency (as when a new crop or animal is 'introduced' or rendered extinct), so the environment will change, and so too will the availability of crops and animals for consumption; food preferences may also change for a variety of reasons, including external cultural influences and food markets.

\section{Regional and Cocal Cuisines}

In the best recognized and most developed national cuisines, as in France, for example, it is common to find 'recognized regional cuisines'. These do not necessarily follow the administrative divisions of the country but are related to particular environmental, economic, social and cultural configurations which have historically given rise to notable cooking cultures or traditions. This means that, in terms of the geography of national cuisine, there may be recognized identifiable regions where a distinctive cuisine is to be found, and there may also be places where there is no particular recognized cuisine or cooking tradition - in other words, blanks on the geographical map.

Ultimately, a cuisine is a social construct - a historical evolving tradition, reproduced over time by the people of a region or locality. This tradition, however, may be copied, in part, by others, wishing to emulate and reproduce the particular tradition elsewhere. In France, as the Larousse Gastornomique puts it, 'the various French provinces have each their particular cuisine. However, it should be observed that the dishes with a regional name are not always authentically and exclusively regional'.

Characteristically, for example, towns and cities will present a variety of cuisines and traditions, derived from their original rural base, and accumulated or congregated together in one place as a result of the movement of peoples and their food traditions, and the emergence of a market or demand for a variety of foods and cuisines. Here, particularly, will be found the most cosmopolitan and sophisticated versions of regional and local traditions; here these traditions of cuisines will be juxtaposed and will jostle each other for attention. Famous cooks, chefs and restauranteurs may arise and become famous for a particular approach to cooking or for a supreme example of a particular regional or local cuisine - even for a particular dish.

But regional and local cuisine is rooted in the small towns and rural areas, where the 'natural' ingredients are available which provide the distinct elements or components of the particular cuisine. Thus, certain mountain areas may 
become known for cooking based on the wild game to be found there; areas with good rivers may become known for their focus on fish; coastal regions for their sea-food; and so on. Geology and soils, wild flora and fauna, climate, patterns of rainfall, seasonal variations, crops grown - all of these will play their part in defining the authentic regional or local cuisine.

There are certain regions well-known for their sea-food, others for their fine game, others for the way in which the sauces are developed, others for their robust stews - a combination of local ingredients and local traditions constitutes the local cuisine. Some have become famous, others remain little known, except to the locals. The same is the case, in France and some other countries, for alcoholic beverages, such as wines and beers. Certain regions in France (and Germany and Italy, and increasingly other countries including those of the 'New World' and Australasia) are well-known, indeed famous, world-wide for their wines. Champagne, for example, has a world wide reputation for its sparkling wines. The cooking in this region is excellent, according to the Larousse Gastronomique, but its repertoire is rather limited, and depends largely on its charcuterie (pork sausages, hams and knuckles, and pigs trotters), fresh water fish (notably carp, pike, trout and salmon), mutton and game. One beverage virtually defines the gastronomic region.

Even within recognized regions there are 'local' cuisines, based on local specialities, which may not be found throughout the region. This may because of the distinctive local flora and fauna, or because of the historical traditions that have developed there. As regards wine, for example, these vary enormously, even within a region, according to the specific rainfall and other climate and environmental features, the lie of the land and nature of the soils in particular. Thus each identifiable wine will have its locality and even its 'terrain' or 'micro-environment', which provides its distinctive character, and there will be 'good' years and 'bad' years, as regards the grape harvest and the quality of the wine produced.
So a local, as well as a regional and national cuisine depends on the environment, the historical cooking traditions and the available ingredients. But all of the potential ingredients actually available in the locality or region may not be in effect of interest to the cooks and consumers of local food. For cultural factors ultimately determine what is seen to be available - what is considered of good quality, what is considered a reasonable food stuff or ingredient and what is, frankly, considered inedible uneatable.

\section{Taboos and Prohibitions}

What is considered to be 'available' may vary, depending on the rules prevalent in any particular society or social group as to what may be legitimately consumed, when and by whom. Many cultures have complex rules - involving outright prohibitions (never to be consumed) and contingent prohibitions (to be consumed only at some times or by some people) and also recommendations (what is considered especially 'good' at particular times and for particular people) - as to what may or may not, should or should not, be consumed, when and by whom.

Most discussion of such 'rules' relating to food preparation and food consumption focuses on taboos or prohibitions, often ignoring the great complexity of many systems of 'food' classification and particularly the common identification of a hierarchy of foods (from mundane to special) and the wider significance (practical and symbolic) of different kinds of food. Most food taboos involve the meat of a particular kind of animal, including mammals, rodents, reptiles, amphibians, bony fish, and crustaceans. Some taboos are specific to a particular part or excretion of an animal, while other taboos forbid the consumption of specific plants, fungi, or insects. Some taboos relate not so much to the kind of animal or plant but to the method of killing and preparation (as in kosher or halal).

The strength and scope of food taboos may vary. For example, some animals are strictly forbidden or 'taboo' at all times; others may be regarded as 'dirty' or undesirable, but may be eaten under certain conditions; some animals may be killed, or plants uprooted, at certain times of the year, 
or under specific defined circumstances. Some foods may be actively encouraged or prohibited at certain times of the year (eg 'the hunting season'), during certain festivals (e.g. Dashain, Christmas, Lent etc), at certain times of life (e.g. pregnancy), or to certain classes of people (e.g. priests), although the food may otherwise be generally permissible.

The system of rules relating to food preparation, production and consumption in any given society is almost always linked to a wider system of classification of what might be termed the social environment' - including social relations, the 'natural' world and the 'supernatural'. Thus they stand at the centre of the social geography - or cosmology, in the widest and most inclusive of senses - of any society.

In the Book of Leviticus (in the Bible), as Mary Douglas reminded us (in her book Purity and Danger), there are complex rules set out as to what animals and plant products may be eaten by the Jews and the early Christians and which may not; The Qur'an also refers to these food rules - these cultural (in this case 'religious') prescriptions and proscriptions defined the range of what was considered edible and what was considered not-edible or inedible (the two being sometimes importantly different - not to be eaten and uneatable). But the rules need not be underpinned by 'religious' systems of beliefs and practices.

In many societies there are distinctions made between 1) animals that are wild and not eaten, 2) animals that are wild and eaten - game), 3) animals that are domestic and eaten, and 4) animals that are domestic but not eaten - pets. Some wild animals may be regarded as vermin and killed as such, others may be hunted and killed for sport, but not eaten (like the fox, or the lion); others may be hunted, snared or otherwise caught for sport and eaten (these include in Britain, rabbit, hare, deer, partridge, pheasant, wood pigeon, quail, and several kinds of seafood - including fish and shell-fish). Some domestic animals may be eaten (such as calves, bullocks, pigs, sheep, lambs, turkeys, capons, cockerels, chickens, farmed fish); others may not (eg dogs and cats, and any animal defined as a 'pet').
There may also be ambiguous creatures, which fit uneasily into the prevailing categories, particularly as the categories change. Crow, eel, singing birds, were all historically widely eaten in Britain; prawns, octopus, squid, etc have recently entered the British menu - as have many food stuffs from around the world. For the Romans, 'mice cooked in honey' was a recognized delicacy; few, probably, would wish to emulate them today.

\section{Food Preparation - First Stages}

Levi Strauss made the point that in virtually all societies, important distinctions were made in the local 'cuisine' between different kinds of food and different food preparation processes. His basic distinction in his book Le Cru et le Cuit (The Raw and the Cooked) was between 'raw' and 'cooked' food. In some cultures, however, even 'raw' (ie un-cooked') food may be prepared and in some way processed, thereby transformed from a plant or fish or animal product into a foodstuff. Indeed, food preparation is the process whereby the 'raw' product is transformed into food.

The first stage of food preparation, at least as far as meat is concerned, is the slaughter of the animal or animals selected. How they are killed is often a matter of concern. For many cultures (including particularly Buddhism) the taking of life, if not forbidden, is regarded with great seriousness and the method of dispatch of the living creature is carefully defined. Even if the technology is not capable of ensuring 'a quick kill' - something of concern in most contemporary 'Western' societies - the death of the animal is often accompanied by a formal recognition of some kind of the significance of its passing. In Jewish and Muslim societies, in which, for example pigs are forbidden in any case, the method of killing other permitted animals (sheep and goats, chickens etc.) is of major importance - meat not killed according to the approved method and technique may not be eaten. The meat must be kosher or halal.

The slaughtering of the animal, or the uprooting, collection or picking of the vegetable, plant or fruit begins the process of its transformation from a 'natural' state to something different. It can no 
longer go on living, even if it may retain certain features of a living thing for some time, if 'preserved' in, say water, for plants. In the case of animals, this process begins at once. The animal's 'corpse' (dead body) is progressively re-defined. One of the first procedures is to remove the outer 'layer' or skin, after which the 'corpse' becomes a 'carcass'. It remains, however, the carcass of a cow, sheep, pig or whatever, until it is cut into pieces. Then it becomes 'meat'. In English, in the case of a pig, it becomes 'pork', in the case of a calf, cow or bullock it becomes 'beef', in the case of a lamb or sheep it becomes 'lamb' or 'mutton'.

For poultry (an interesting collective noun for all domestic birds reared for slaughter and consumption), the term carcass is rarely used for the complete dead bird - rather it is referred to at this point as 'the bird'. When prepared and ready for cooking, they are referred to still as 'birds': cookery books may say, for example, 'put the bird in the oven', or, more specifically, 'put the turkey into the oven'. When cooked and dismembered or 'cut up' (carved' is the term used for cutting into pieces after cooking), the bird becomes 'meat': a hen or cockerel becomes 'chicken', a duck becomes 'duck', a goose becomes 'goose' and so on. Individuality is destroyed; live creatures become 'meat', to be classified by 'cut' (breast, wing or leg) or type of meat (dark or light).

This kind of conceptual transformation, involving a process of 'anonymization', is less significant in the case of fish, particularly when the identity, the kind of fish is reduced by being cooked with other ingredients (as in 'fish pie); although when presented whole or as a fillet it may be referred to by its name (ie fillet of sea bass, grilled trout, etc.). Such a process of transformation rarely takes place in the case of root or leaf vegetables, or fruit - a living carrot and an uprooted carrot are both 'carrots'. But, when prepared and cooked it also tends to lose its indefinite article and is transformed into 'carrot', or becomes entirely unrecognizable in a mixture or dish.

In some cultures animals may be killed by the very process of cooking - for example lobsters and crabs are commonly tossed alive into boiling water and then cooked until 'ready'. In this process, 'a crab' becomes simply 'crab', and 'a lobster' simply 'lobster'. Locusts, which are widely consumed in parts of sub-Saharan Africa, experience a similar fate, in so far as they tend to be fried alive and salted. This is relatively rare, most peoples preferring to ensure the transformation of the living creature into and 'anonymous' edible form first.

\section{'Cuts'}

The carcass of a dead animal, whether hunted to death or domestically slaughtered, is usually divided up, partly for convenience - a beef carcass can be very large, and cumbersome, but partly to convert it into 'meat'. In some cultures, specific parts are given to specific people, even at this stage - particularly in situations where the animal has been either owned collectively or killed collectively, in a hunt, for example. Different societies prize different parts. In some hunting societies, the heart and kidneys and liver may be eaten shortly after the kill, while still fresh and 'uncooked'; in some cases they are removed, grilled or roasted, and eaten. Sometimes these parts are eaten at once, before the rest of the carcass is cut up, sometimes allocated and taken home to be processed and prepared further.

Different societies recognize and make use of different parts of the animal and convert them to identifiable 'cuts'. In many cultures, the division of parts remains fairly arbitrary, even when a specialist is involved. But in some cultures, particularly where a professional butcher slaughters and then prepares the meat - or a fishmonger prepares the fish - for householders, the next stage of the preparation has become standardized. In this case, the meat will be cut up into defined parts, each with its specific standard name.

In England, these parts are actually known as 'cuts' of meat. The major cuts regularly include, for beef: neck and clod, chuck and blade, thick rib, thin rib and fore rib, brisket and shin, sirloin and flank, rump, silverside and topside, thick flank and leg. In the USA the major cuts are: chuck, brisket and shank, rib and plate, flank, 
short loin, sirloin, tenderloin, top sirloin and bottom sirloin, round and shank. Further distinctions may be made, for example, again for beef: head, scrag end, neck, shoulder, ribs, belly, topside, silverside, steaks (sirloin, rump and fillet), loin, haunch, skirt, shank, hock, shin, leg, tail etc. In the case of pork, somewhat different and additional cuts are recognized, which include ham, bacon (both of which may be cured for longevity) as well as the range of pork ribs, chops, steaks, belly and so on. In addition to these cuts or additional items, is the 'offal', which includes the internal organs brain, heart, liver, kidneys, stomach, intestine, tripes - as well as some external organs - testicles (sweetbreads), udders etc. Other now-less-used parts include: tongue, eyes, brain, cheek (pig), trotters (pig) and heel (cow).

In European - and in some other national cuisines different cuts of meats require different preparation and cooking, and are eaten on different kinds of occasion. Offal, in particular, is today regarded with suspicion by most but the most adventurous or traditional cooks - and even within the category of offal, there is a hierarchy from the liver, kidneys, heart, sweetbreads (testicles), tripe(s), udders, intestines (chitlings), and stomach. Historically, these parts of the animal were often regarded particularly highly and eaten as delicacies; certainly they tend to be highly flavoured when compared with more currently orthodox 'cuts'.

In Western societies, particularly in North America and Britain, the processing of meat has progressively privileged certain cuts and items and re-defined others, marginalizing them, and giving them the status of 'low grade' or even quasi-taboo food. In general, the 'cleaner' cuts, with least reference to or identification with the original animal, are privileged. The additional items identified above are rarely eaten nowadays, while it is increasingly difficult to obtain offal. Worries about obesity and the medical conditions associated with a 'bad' diet has led to the progressive reduction of the amount of fat left on cuts from the butchers, while it is also harder to find meat 'on-the-bone'. The rest of Europe, particularly France and the Mediterranean countries remains less concerned, but similar tendencies, 'to simplify and clean up' meat, are becoming apparent there also.

The same tendencies - of processing food stuffs more and more, taking them further and further away from the original animal or food stuff - are apparent in the processing and preparation of other items besides meat. The progressive removal or elimination of certain 'natural' elements and the addition of 'additives' (to enhance flavor or to increase 'shelf life) is part of the industrialisation of food processing and preparation and the transformation of food into a commodity or commodities. Food 'preferences' have often moved in line with this tendency over the last century or so (eg white rice instead of brown; white flour instead of brown or wholemeal). In more recent years, however, there has been a 'backlash' against this tendency and, to some degree, a reversal towards 'authentic', 'real' and 'organic' food and drink.

\section{High and Low Cuisine - Processed Food and 'Home Cooking'}

After the basic food preparation discussed above, further preparation may be relatively simple or relatively complex. A 'simple meal' may consist of a limited number of elements dishes or ingredients - taking a relatively short time to prepare. Today, 'fast food' is a term that tends to identify food that is immediately recognizable, is 'clean and simple' and that takes relatively little time to prepare in the home. Often, however, although it may often involve a relatively short time overall to prepare at home (as in a bought cooked burger or 'fish and chips') it actually involves more time in preparation elsewhere. The ultimate here is the so-called 'ready meal' which needs simply to be heated by the consumer to be ready to eat, but has undergone very complex processes to prepare in the place where it is initially 'manufactured', and took a relatively long time to prepare-hence 'prepared meals' or 'ready meals'.

Generally simple preparation is for simple social occasions. More complex preparation is required for more complex meals, consisting of more complex and more elaborate dishes and, usually, more august or special company. In general, many cultures identify a 'higher' and a 'lower' 
cuisine, the former comprising relatively complex dishes and a relatively complex and elaborate overall presentation of the dishes, the latter simpler dishes and a simpler overall format. In some cultures there is a distinctive 'court' cuisine or tradition of cooking, which represents the highest form of culinary art available. In his book on Indian cooking, for example, Michael Pandya, distinguishes between pukka and kacha Indian cuisine or cooking - the former being 'proper' and the latter being 'simple' cooking. The same distinction is made widely across the world.

Another distinction, closely related to the preceding one, is that made between 'home cooking' and 'high cuisine', between the forms of preparation, cooking and serving commonplace in the home, on the one hand, and those found in those restaurants where there is a 'chef' rather than a 'cook' and the food is expected to be 'entirely different' and superior, on the other. It is often said that the best way to get to know and appreciate a particular national, regional or local cuisine is through the 'good home cooking' that characterizes it most effectively; but it is also the case that the 'finest cuisine' can be said to epitomize 'the best' of a national or even regional tradition.

But it should be appreciated that even with 'home cooking' there are differences between the style of preparation, cooking and serving cuisine-associated with ordinary meals for the family, meals for friends and relatives, meals for special occasions, and feasts, to identify four at the very least. 'Every day cooking' is minimalist in every sense and might be termed 'mundane' or 'routine'. It is what is routinely prepared for the members of the household on a normal working day. If a wider circle of relatives and/ or friends are involved, it will, generally, be somewhat more elaborate; if it involves 'guests' beyond the circle of family and friends it will be more elaborate still and may involve a change of 'style' (involve different forms of preparation, production or cooking and presentation or serving), and generally a larger amount will be provided per person. Meals for special occasions are again of a different order and may resemble meals prepared for guests. Finally, there are feasts, prepared for festivals and life-cycle ceremonies, which will generally show the greatest degree of elaboration and complexity in preparation, production, presentation and serving.

\section{Food Preparation - Second Stages}

Most cuisines draw upon a range of different modes of food preparation and of cooking which may be utilized in various combinations, depending on circumstances. The most basic food preparation involves 'cleaning' the food (meat, fish or vegetables) - this may involve washing (fruit and vegetables and leaves), taking off the 'outside' or 'surface' (peeling, removing the skin, cutting off the fat, scraping, etc.) or removing various undesirable elements from within (de-boning meat, de-gutting and filleting fish, de-veining leafy vegetables, 'coring' fruit and removing pips etc.) or sieving (flour) to remove large and undesirable particles. This process of 'cleaning' may be supplemented by 'trimming'. Assuming that the meat is already in the form of a basic 'cut' (although it may not be, in which case it will need to be 'cut' according to the dish and meal to be prepared from a larger part of the carcass), it may need to be further 'cut and trimmed' - the meat may have the fat removed, or it may be cut into smaller pieces, the fish may have its head and tail removed, the vegetables may be cut into slices, the fruit into segments.

Fish is generally filleted (main backbone removed and other bones removed as far as is practical or else as demanded by the 'quality' of the dish and the meal - although small fish may be cooked whole). Often heads and tails and fins are removed, as are scales. In some cultures, as in Japan, fish in particular may be eaten uncooked ('raw'). This does not mean, however, that it remains unprepared. Indeed, some varieties - delicacies - are, notoriously, poisonous until certain parts are removed. Most dishes of 'raw' fish served in Japan have been at the very least, cleaned and scraped where necessary and cut into appropriate sections or portions. Meat also may be eaten raw, or rather uncooked. Thus, steak tartare, for example, consists of fine cuts of raw beef, minced and served in a distinctive sauce. 
Vegetables may be eaten raw. Leaf vegetables, used for salads, are often uncooked and merely mixed and served with a 'dressing'. Some other vegetables (eg carrots, celery, courgettes may simply be cleaned and cut into slices or sections and served as 'crudites' (literally 'raw pieces' in French), as a preface to a meal or as the accompaniment to a 'dip'. Leaves of some green vegetables and even shoots of members of the onion family may be used, uncooked, as salad, to accompany a meal or a dish, or as a garnish or decoration (as in the case of parsley or coriander leaves).

Fruit is often eaten raw, even in the course of a meal (as opposed to being eaten separately), but is usually 'prepared' in some way to transform it from the original fruit to a prepared fruit again, as with meat, fish or even vegetables to render it less recognizable. It may only be peeled, cut into sections or segments, and arranged on a plate or dish; or it may be processed simply in some way to produce a new concoction constituted of the fruit - mashed banana, chopped pineapple, sliced pear, apricot puree. Simple, but slightly more, processing may occur, when the fruit is mixed with some other ingredient - to make fruit salad, gooseberry fool, mixed fruit puree, etc. In most cultures, particularly where a wider range of other food stuffs is available, fruit is regarded as a complement or supplement to a meal - a dessert at most - rather than a component of a meal, unlike vegetables or pulses, which in vegetarian cuisines may be the only ingredients apart from herbs and spices.

The importance of fruit varies, however, from cuisine to cuisine. In some cuisines (eg Moroccan and Persian), fruit plays an essential part as an ingredient in dishes whose major elements are meat or, to a lesser extent, fish. In this case, more complex processing, involving the cooking of the fruit together with other ingredients may take place. Vegetables may also be made into purees and soups, but these will need cooking and sieving before this transformation takes place.

Once 'cleaning' is complete and 'cutting and trimming' is done, other forms of processing or preparation may take place. These may include procedures that do not involve cooking - such as marinading (meat and or fish), 'smoking' (meat and or fish), 'salting' (fish), 'soaking' (lentils and pulses, dried fruit) or 'tenderising' (ie beating, immersing in vinegar, lemon juice or some other acid, yoghurt, or adding special condiments). Some of these methods also make possible the preservation of foods - eg salting, marinading and smoking. To these should be added drying - which may be applied to meat, fish, vegetables and fruit. Dried foodstuffs may be reconstituted by soaking in some liquid or oil.

Preparation and processing may include the preparation of marinades, pickles, chutneys, pastes, collections of herbs and or spices that will be used in the cooking process at a later stage. A prime example of this in Indian and Nepalese cuisine is the preparation of chat masala (spices for a chat, or snack) or garam masala (literally hot spices), which is a specific mixture of spices widely and commonly used in cooking. In English this may be termed 'a preparation'.

Once the initial preparation and processing is completed, the main task may begin - the cooking. This, at its most basic, involves applying heat to the selected ingredients. This can be done directly, over a naked flame or fire, over charcoal or in the ashes, as in grilling and roasting; or in an oven, where the heat may be direct, as in roasting or baking in a gas or electric oven (or in a pit, where much the same effect may be obtained as in an oven, but without the more or less permanent structure involved with an oven). Heat may also be applied indirectly, with the foodstuff on a griddle, plate or pan of some kind (eg wok), as in searing, pan-frying, stir-frying, either 'dry' or with some fat, butter, ghee or oil. The use of pots and pans - containers that can retain liquid - allows a range of additional cooking methods to be utilized, including boiling (in water), simmering (in stock or some other liquid), pot-roasting (where the meat and or vegetables are cooked in a closed pot which them itself placed in an oven) and stewing.

\section{Towards a Conceptual Framework}

Over the centuries and millennia, there has been a gradual and progressive evolution in the complexity of the technology associated with 
cooking, but also there has been a complex (and not necessarily contradictory) process of development, involving both the emergence of distinct and differentiated local and regional and even national and imperial or international cuisines, and also the exchange and interchange of ingredients, cooking methods and traditions, leading to a mingling and inter-mixture of traditions and forms of fusion.

As a result of these historical processes, it is often possible to recognize and identify distinctive cuisines, but the 'classification' of such cuisines needs to be tempered and qualified on the basis of the recognition that ingredients, methods and techniques, traditions and styles of cooking are cultural forms which evolve and interact with each other over time and space. In a real sense, therefore, the 'cuisine' is a historical and cultural artifact or construct whose features, characteristics and boundaries are environmentally, economically, socially, culturally and historically determined. In so far as the cuisine as a bounded entity is also generally more or less spatially defined (as are cultures and traditions of all kinds) it is a geographical phenomenon.

Most cookery books which discuss the notion of 'cuisine' implicitly identify 'national cuisine' as a conceptual category (although they rarely examine in any detail the process of categorization or discuss the term cuisine as a concept, as I have tried to do in the earlier sections of this article). Thus, in common parlance, as in books on food, there is a tendency to speak of French cooking, Italian cooking, Chinese cooking etc. Good Housekeeping's World Cookery (1962), for example, is largely divided into chapters on the basis of individual countries, although sometimes - where the identification or recognition of national cuisine is poorly developed - they are grouped into larger entities, as with the Middle East, the Far East (which includes India, Pakistan and Ceylon). These larger 'food traditions' also, however, are based on identifiable common characteristics whether ingredients, food preparation and processing, styles and methods of cooking and forms of presentation - even if these may not be clearly defined.
Thus, Claudia Roden initiated Western readers into the food traditions of the Middle East with her Book of Middle Eastern Food (in 1968) and Elizabeth David, at a slightly earlier period, was able to introduce western Europeans to the food traditions of the Mediterranean with her Book of Mediterranean Food (first published in 1950). More recently, however, Claudia Roden has identified more specific national and cultural food traditions, in her books on Jewish food, Moroccan, Turkish and Lebanese food, while Elizabeth David has written extensively on French, Italian, Spanish and Portuguese food. In the last two or three decades, a multitude of cookery books dealing with food from all over the world has been published. The identification of regional/international and national cuisines as food traditions developing in specific moreor-less defined geographical areas and in constantly evolving historical socio-economic and cultural contexts, with specific ingredients and with distinctive approaches to and methods of preparation, processing, production and presentation, is still, however, very generalized and surprisingly little explored.

The focus is still very much on recipes and the taste of the food itself rather than on the historical, social and cultural context of its preparation, production and presentation, although some writers, such as Claudia Roden, do provide more of a context than most other food writers. In the 'great French tradition', little recognition is given to any other cuisine. In the Larousse Gastronomique, for example - a classic source of information on the French approach to food and cooking - 'Italienne' is described as 'a name given to various dishes made of meat, poultry, fish and vegetables, in all of which, finely-diced or chopped mushroom are used'. It is as though the only defining characteristic of Italian cuisine is the use of chopped mushrooms. Indian cuisine is, perhaps not surprisingly, not mentioned at all.

In the Good Housekeeping's World Cookery, Indian cooking is mentioned under 'The Far East', together with Pakistan and Ceylon. This was published in the 1960s, at about the same time as Claudia Roden's Book of Middle Eastern Food, when 'Indian cuisine' was still little known in the West and a distinctive British-'Indian' 
tradition was being invented by expatriate Bangladeshis for the most part. The section of recipes is prefaced by a short introduction 'some notes on Indian cooking'. It notes that, by the 1960s, 'many Indian condiments can be bought here (in Britain), including mango chutney, chilli paste and Bombay duck'. It states that 'curry powder and curry paste can be bought in prepared form', but also emphasizes that 'you can obtain even better results... by using the fresh spices of which they are composed: these are chiefly coriander, turmeric, cumin seed, ginger, mace, cloves, cardamom and pepper' and recommends that 'spices should be freshly ground if possible..' It goes on to remark that 'the cooking of many curries starts with the frying of the spices'. The idea clearly developed here is that there are distinctive aspects to 'Indian cuisine', but there is no reference in these 'Notes' on differences between different 'Indian' cuisines, despite the size of the sub-continent and variety of different forms of preparation, cooking and presentation to be found there. 'Indian cuisine' is presented as an undifferentiated whole.

In France, by contrast, and in the writing on French cuisine, it is common to find specific 'regions' identified as gastronomic regions or as having their own distinctive cuisine, since at least the $18^{\text {th }}$ century. The Larousse Gastronomique (Paul Hamlyn, London 1961) remarks that the various French provinces have each their particular cuisine' while warning that 'it should be observed that dishes with a regional name are not always authentically and exclusively regional'. It goes on to identify specific regional cuisines, including Alsace, Anjou, Artois, Auvergne, Bresse, Brittany, Champagne, Lorraine, Languedoc, Lyon, Normandy, Provence etc. but on the basis largely of the significance of the cuisine rather than simply on the basis of a geographical or administrative region. So, Lyon, though a small region comprising only two departments 'deserves a place in the first rank for its excellent cuisine. Lyons can in fact be regarded as the gastronomic capital of France'.

\section{Indian Regional Cuisine}

Even today, Indian cookery books and books on Indian cuisine tend to amalgamate recipes without regional distinction, preferring the more conventional division into types of dish according to ingredients (meat, fish, vegetable) or into elements of the meal in typical French banquet sequence (soup, hors d'oeuvre, fish, roast meat, vegetables (entremets) entrée, poultry, salad, cheese, sweets or desserts). When they do distinguish regions, it is common to find Indian cooking as a whole divided roughly into major geographical regions - northern, eastern, southern, central and western - each of which is 'characterised' in extremely broad or sketchy terms.

Thus, Michael Pandiya in his Complete Indian Cookbook (1996) identifies a 'northern cuisine' which he equates with Kashmiri and Punjabi cuisines. He suggests that the former specialises in friend meats and curries without onion and garlic, the latter in tandoori, paraunthas, sweets and delicacies. Very generally, he suggests, dishes are accompanied by bread made of wheat flour rather than rice, although 'liquid dishes' (with sauces - ie 'curries') do tend to be served with rice. He speaks, vaguely, of the influence of the Mughals. He also identifies an 'eastern region' including Bengal and Bihar. Here there is 'a considerable use of fish'. Rice is central as an accompaniment to all dishes. Cooking is generally in mustard oil, and favourite spices include mustard, cumin, anice and fenugreek. Great sweets and savouries are also characteristic of the eastern cuisine. The central region is said to be 'complex and fine' and to be dominated by Mughal style cooking. But little more is provided.

The western region includes Mumbai and Goa. Goan food is described as 'usually hot, with lots of coconut milk, boiled into thick or thin gravies, and use of tamarind juice, as in south India'. Spices are 'usually ground before use, rather than fried or roasted whole before crushing'. Long slow cooking is characteristic. South Indian food is 'largely vegetarian with rice the main accompaniment to a meal'. There is widespread use of mustard oil; coconut, tamarind, and coconut milk widely used. Curries are thin and hot (eg vindaloo), particularly around Madras. 
And paper-thin papadoms are a speciality. Meat, when used, 'is rubbed with spices rather than marinaded as in the north'.

Khalid Aziz, in his earlier Indian Cooking (1983) also identifies the same five main regions, but refers also specifically to the Deccan (Hyderabad) and the use of coconut. He points out that dhansak (which involves a dish combining meat with lentils is a Parsee dish ('dhansak' means 'wealthy'). The northern cuisine is 'characterised by meat cooking. There is strong Mughal influence and cooking is tandoori style with naan and rotibreads'. The Goan gol guppa (a snack) is mentioned specifically. Meera Budhwar also recognizes five regions of Indian cuisine, but identifies Kashmir and Rajasthan as 'the northwest' - and sees it as characterized by meat cooked in a sealed clay pot-dum. She recognizes also the cuisine of Bengal, that of Lucknow, that of Mumbai in the west, and that of Kerala, Cochin - in the south.

So even with respect to Indian cuisine, which has been far more widely discussed and explored than Nepalese cuisine, there is still a very crude regionalization and identification of different distinctive cuisines. Individual recipes, however, sometimes indicate the regional origin and background. Thus, even World Cookery identifies a number of dishes from different parts of the country: Some of the recipes provided clearly indicate their origin in a more specific cuisine, thus 'Bengal curry', 'Calcutta Beef Curry', 'Madras Meat Curry', 'Bengal Chicken Curry', Madras Chicken Curry', 'Deccan Duck Curry', 'Deccan Meat Burtas', 'Madras Chicken Pellao', and 'Deccan Lentil Curry'. But the warning given by the Larousse Gastronomique applies here also: 'it should be observed that dishes with a regional name are not always authentically and exclusively regional'.

Most so-called 'Indian restaurants', in Britain at least, present an 'all-Indian menu', usually undifferentiated as to the region or locality in which the particular meal or dish is a part of the traditional cuisine, but often divided as to the main ingredient (meat, chicken, vegetable), cooking style (tandoori, curry, biriyani, dry or with sauce/gravy) and degree of 'hot-ness' (as defined by the amount of chillies and pepper used). Again, sometimes, the name of the particular dish is associated with the traditional cuisine and place of 'origin' - as in Punjabi chicken, meat Madras, etc. On the other hand, as regards the identification of an 'authentic' Indian cuisine, it is interesting to note that most of the 'Indian' restaurants in Britain are in fact run by Bangladeshis and that the cooks may be Indian, Bangladeshi, Nepalese or, more rarely, Pakistani.

During the last five years in Britain, there has been a growing tendency for restaurants to advertise 'authentic Indian and Nepalese food'. It is still rare, however, to find a restaurant relying simply on Nepalese food and the Nepalese cuisine. It is also difficult to find Nepalese cookery books or even collections of recipes, outside Nepal itself. In part, this is because little attention has so far been paid to the features and characteristics of Nepalese cuisine. We shall attempt to remedy this in the second part of this article.

\section{References}

Aziz, Khalid (1983). Indian Cooking, Perigree.

Budhwar, Meera (1992). The Complete Indian Cookbook.

David, Elizabeth (1950). A Book of Mediterranean Food.

Douglas, Mary (1972). Purity and Danger: An Analysis of Concepts of Pollution and Taboo.

Good Housekeeping (1972). World Cookery. London: Ebury Press.

Levi Strauss, Claude (1969). The Raw and the Cooked. London: Jonathan Cape.

Pandiya, Michael (1996). Complete Indian Cookbook, Chancellor.

Roden, Claudia (1968). The Book of Middle Eastern Food. Penguin Books. 